\title{
Testing and Diagnostics of Carbon Polymer Composite Materials by Radio Wave Method Used in Aircraft Construction
}

\author{
German V. Dmitrienko ${ }^{1, a^{*}}$, Dmitry V. Mukhin ${ }^{1}$, Aleksandr A. Fedorov ${ }^{1}$ \\ ${ }^{1}$ Ulyanovsk Technical University. Institute of Aviation Technologies and Management, 13A, \\ Sozidateley Avenue, Ulyanovsk, Russia, 432072 \\ admitrienko.german@yandex.ru
}

\section{Keywords: Polymer Composite Materials, Carbon Plastics, Dielectric Constant}

\begin{abstract}
The article is devoted to the applicability of radio wave methods of testing and diagnostics of aviation composites for newly launched products. The description of the measurement process is also considered by the method of integral equations.
\end{abstract}

\section{Introduction}

Recently, fundamentally new types of radio-absorbing structural composite materials (CMs) for aviation purposes have appeared, such as: conductive polymers, carbon nanotubes, nanostructured CMs. In electrodynamics, such materials are classified as low impedance -polymer composite materials (PCMs), which are characterized by large values of the relative permittivity $\varepsilon$ and the tangent of the dielectric loss angle $\operatorname{tg} \delta$ simultaneously. The carbon-fibre-reinforced plastics (CFRPs) are widely used in aviation technology as structural materials for creating loadbearing structures, and as radio engineering materials: to reduce the effective reflective surface of aircraft, to protect radio engineering systems from powerful electromagnetic radiation.

In order to increase the accuracy of processing the measurement results and control the characteristics of the product by its dielectric parameters, a more rigorous from the electrodynamic point of view, a mathematical method, the method of integral equations (MIU), is used [1-5]. The procedure for drawing up integral equations is carried out through surface currents induced on the surface of the PCM sample and the metal structure of the waveguide sensor by a probing electromagnetic wave. The dielectric parameters of the PCMs and the characteristics of the reflecting surface are set through the surface impedance. The information parameter is the reflected wave, and the measuring problem is reduced to the problem of exciting a circular waveguide by currents induced in the PCMs by the field of the incident wave. The measurement results are processed by numerical methods. The methodology of drawing up integral equations for bodies of revolution is given in [1-5]. On its basis, a multilayer model of a PCMs sample is compiled.

$$
\begin{aligned}
& E(p)=\int_{L} \xi^{E}\left(p, q, \varepsilon_{a}, \mu_{a}\right) J^{E}(q)+\xi^{M}\left(p, q, \varepsilon_{a}, \mu_{a}\right) J^{M}(q) d l, \\
& H(p)=\int_{L} h^{E}\left(p, q, \varepsilon_{a}, \mu_{a}\right) J^{E}(q)+h^{M}\left(p, q, \varepsilon_{a}, \mu_{a}\right) J^{M}(q) d l,
\end{aligned}
$$

A model of a PCM sample with a rough surface (RS) is represented as a multilayer structure consisting of homogeneous layers, the number of layers is determined by the discrete partition in $\varepsilon$. Integral equations for a multilayer structure are based on the equations obtained earlier (1) and 
(2) for each layer, using both of its boundaries. The method of drawing up integral equations for a multilayer medium is carried out as for a single layer, taking into account the distribution of currents at the interfaces. The compound is carried out according to the boundary conditions of Shchukin-Leontovich since PCMs have $\varepsilon, \mu$ - electrical and magnetic permeability and $\sigma$ conductivity. The sample is divided into $N$-th number of layers; their boundaries have no common points. Each layer can contain third-party sources; the layers are numbered in the direction from the observation source into the interior of the PCM sample. Equations are drawn up, for layers K and $\mathrm{K}+1$ having a common boundary $\Sigma_{\mathrm{K}}$ using (1), (2), the equivalence theorem is applied to the K-layer. The normal at the interfaces between the layers is directed towards the primary sources.

For the K-layer, the equations are:

$$
\begin{gathered}
\frac{1}{2} E_{K}(p)=\int_{\Sigma_{K}}\left[\xi^{E} J_{K}^{E}(q)+\xi^{M} \widetilde{J}_{K}^{M}(q)\right] d \sigma_{q}+\int_{\Sigma_{K-1}}\left[\xi^{E} J_{K-1}^{E}(q)+\xi^{M} \widetilde{J}_{K-1}^{M}(q)\right] d \sigma_{q}+E_{K}^{\Pi}(p), p \in \Sigma_{K} \\
\frac{1}{2} H_{K}(p)=\int_{\Sigma_{K}}\left[h^{E} J_{K}^{E}(q)+h^{M} \widetilde{J}_{K}^{M}(q)\right] d \sigma_{q}+\int_{\Sigma_{K-1}}\left[h^{E} J_{K-1}^{E}(q)+h^{M} \widetilde{J}_{K-1}^{M}(q)\right] d \sigma_{q}+H_{K}^{\Pi}(p), p \in \Sigma_{K}
\end{gathered}
$$

Integral equations are written in matrix form for the $\mathrm{K}$-layer, similarly written for the $\mathrm{K}+1$ layer, the integrals are taken over the surfaces $\Sigma_{\mathrm{K}}$ and $\Sigma_{\mathrm{K}+1}$ as (3).

$$
\begin{aligned}
& \frac{1}{2} E_{K+1}(p)=\int_{\Sigma_{K+1}}\left[\xi^{E} J_{K+1}^{E}(q)+\xi^{M} \widetilde{J}_{K+1}^{M}(q)\right] d \sigma_{q}+\int_{\Sigma_{K}}\left[\xi^{E} J_{K}^{E}(q)+\xi^{M} \widetilde{J}_{K}^{M}(q)\right] d \sigma_{q}+E_{K+1}^{I}(p), p \in \Sigma_{K+1} \\
& \frac{1}{2} H_{K+1}(p)=\int_{\Sigma_{K+1}}\left[h^{E} J_{K+1}^{E}(q)+h^{M} \widetilde{J}_{K+1}^{M}(q)\right] d \sigma_{q}+\int_{\Sigma_{K}}\left[h^{E} J_{K}^{E}(q)+h^{M} \widetilde{J}_{K}^{M}(q)\right] d \sigma_{q}+H_{K+1}^{I I}(p), p \in \Sigma_{K+1}
\end{aligned}
$$

In order to obtain excitable currents on the surface, equation (3) is conjectured vectorially on the normal $\varepsilon_{K+1}$, but (4) on the normal $\varepsilon_{K}$, then equations (3) and (4) are added.

In the process of describing the RS $\varepsilon$ will change from layer to layer, but $\varepsilon$ will be constant inside the PCM sample. Integral equations inside the layer will be simplified. In the description of the reflection process, one can replace the internal structure with one layer, and in the description of the process, the transmission can be replaced by one layer of finite thickness. An integral equation is drawn up for each layer, for the boundaries $\Sigma_{-1}$ и $\Sigma_{N+1}$ there are no integral equations, as well as the boundaries themselves. At the outer boundaries of the PCM sample, the ShchukinLeontovich boundary conditions are applied. Equations are stitched inside the polymer composite materials. The multilayer model described by the International Innovative University is convenient for describing, predicting and modeling the temperature characteristics of PCM.

\section{Discussion of the results}

Integral equations are solved by numerical methods. The most common is the projection numerical method (Galerkin method) [1].

According to the given theory, a control was carried out and the value of the relative dielectric constant of the PCM sample was determined, material U-PU $\varepsilon=950 \pm 30 \%$ and the tangent of dielectric loss angle $\operatorname{tg} \delta=1,3 \pm 40 \%$, having a reflection coefficient modulus of 0.94 [6].

The main problem of constructing simple design engineering formulas. Difficulties are caused by the surface roughness (RS) of the measured material. For conducting polymers, it can be of 4- 
8 classes, which significantly introduces uncertainty in the final result. The final result is the complex dielectric constant $\underline{\varepsilon}$ of the PCM sample. We measured PCM samples with a rough surface, the level of which was 5-7 microns, which corresponds to an additional phase incursion of the order of $0,1^{\circ}$ in the three-centimeter wavelength range. The RS level of the reference standard, relative to which the control is carried out, is 1 micron.

The assessment of the level of surface roughness of the measured sample must be carried out in the instrumental part - the introduction of additional intermediate exemplary measures, as well as in the methodical part - the introduction of corrective additives, taking into account the surface roughness of the measured PCM sample. Other types of uncertainties are present in traditional methods and ways to measure and can be considered known.

\section{Analysis of the influence of the RS of PCM on the control results}

To control the dielectric characteristics of PCMs, first of all, the quality of their technological preparation, that is, the roughness of the measured surface, will be of interest. Methods for measuring RS of products regulated by GOST 2789-73 and GOST 27964-88 [7], turned out to be unsuitable for controlling the surface roughness of PCM products, because measurement of RS is carried out at some points and in small areas. In addition, the surface condition of PCM products is characterized by the following features: characteristic color, porosity, cracks, texture of the reinforcing filler, cavities, chips, sooty formations, and roughness [9-11]. Some of these features relate to the structural features characteristic of a given composite material, the other is due to the modes of mechanical processing of the product, the rest of the features refer to the technology of manufacturing the product from this composite. The RS is an initial requirement for the technological process of surface formation, and it is directly or indirectly related to various indicators of its quality. The control of the RS of PCM products is the most difficult due to the peculiarities of the PCM structure. So, for example, the structural features of the surface of carbon composite materials are: pores, shrinkage cavities, pile of fibrous fillers, coke grains up to $3 \mathrm{~mm}$ in size, etc. The RS, in the form of inhomogeneities, with sizes from $0.5 \mathrm{~mm}$ to $3 \mathrm{~mm}$. For example, in the technical documentation for a product made of carbon-containing PCM, the RS parameter is usually set by the numerical characteristic $R_{z}$, which is determined on the basis of a profilogram. The RS of PKM can vary from 1 micron to $0.5 \mathrm{~mm}$, so that the uncertainty of measurement of the $\mathrm{RS}$ will be determined by the uncertainty of the measured device. PCM samples for measurement are checked for conformity to the plane, according to the RS, in terms of geometric dimensions, in homogeneity, in the absence of mechanical damage, etc. The uncertainty in the manufacture of samples is $\pm 0,005 \mathrm{~mm}$.

In the process of preparing PCM samples for measurements, they have an unremovable RS, which leads to additional instrumental and methodological uncertainties. As noted earlier, the uncertainties caused by the RS of the measured PCM sample are predominant. The uncertainty of the phase of the reflected signal caused by the displacement of the reflection plane is large, comparable in magnitude with the value of the instrumental uncertainty in measuring the phase. Since in dielectric measurement it is considered that the plane of reflection from the dielectric material coincides with the plane of contact with the measuring sensor, the RS of traditional materials $(\varepsilon<100)$ is not taken into account. In reality, when measuring PCM with the RS, the question arises about the location of the reflection plane. Depending on the nature of the irregularities of their height on the surface, the geometry, the reflection plane will be above or below the median surface of the PCM irregularities. The RS model, presented in the form of a multilayer structure, makes it possible to verify this numerically. Therefore, it is necessary to carry out an analysis to take it into account in the process of calculating of $\varepsilon$. 
There is a known method of representing the RS through an intermediate layer, in which $\varepsilon$ changes, along a gradient deep into the material. This method is complicated in the process of numerical implementation. A more accurate representation of this rough layer is represented as a multi-layer structure in which each layer has its own average value of $\varepsilon$.

This method of representing RS gives the best accuracy, the disadvantage of this method is the need for accurate knowledge of the geometry of the relief of a rough surface in order to determine the average value of $\varepsilon$ in each layer.

To improve this technique, it is proposed to represent the WB in the form of a multilayer structure. From a mathematical point of view, a WB consisting of sublayers of thickness $d_{i}$ - can be considered with an average value of $\varepsilon$ calculated by the Lichtenecker equation [12] for complex dielectrics

$$
\varepsilon \Sigma=\varepsilon_{0} V_{1}+\varepsilon_{1} V_{2}, \quad V_{1}+V_{2}=1
$$

where $\varepsilon_{0}$ is the dielectric constant of the consolidated space, $\varepsilon_{l}$ is the dielectric constant of the PCM, $V_{1}$ is the filling factor of the sublayer with the consolidated space from the entire volume, $V_{2}$ is the filling factor of the sublayer of the PCM from the entire volume of the sublayer. As a result, we get an intermediate layer representing the RS between free space and PCM, consisting of several thin sublayers in which $\varepsilon$ changes according to a certain mathematical law.

The distribution law $\varepsilon$ over the layer is mathematically represented as a sum of sublayers $\sum_{i=1}^{N}\left[\varepsilon_{1} V_{1 i}+\varepsilon_{2} V_{2 i}\right]$, where $V_{1 i}, V_{2 i}$ are the filling factors $i$-sublayer from the entire volume. The RS of PCM is presented in the form of a multilayer structure, in which each of the layers has a different filling of the PCM. A mathematical model of a complex RS is presented, which is divided into several sublayers, representing it in the form of a plane-parallel structure. Each sublayer of such a structure is characterized by a different coefficient of filling the layer with a dielectric, and its $\varepsilon$ is considered averaged over the layer, while the sublayer is considered homogeneous and isotropic. The reflection of a plane wave from such a structure is calculated through a transmission matrix describing each sublayer. The number of layers is selected depending on the level of the RS and the distribution of irregularities was uniform over the selected discrete $\Delta \varepsilon$.

Each sublayer is described through a characteristic matrix [8], which is a transmission matrix. The characteristic of each layer is represented by the transmission matrix of the sublayer depending on the filling factor of the PCM sublayer. So for the $i$-sublayer of the transfer matrix $M_{i}$ is equal to

$$
M_{i}=\left[\begin{array}{cc}
\operatorname{ch}\left(j k d_{i} \sqrt{\varepsilon_{i}}\right) & \frac{1}{\sqrt{\varepsilon_{i}}} \operatorname{sh}\left(j k d_{i} \sqrt{\varepsilon_{i}}\right) \\
\sqrt{\varepsilon_{i}} \operatorname{sh}\left(j k d_{i} \sqrt{\varepsilon_{i}}\right) & \operatorname{ch}\left(j k d_{i} \sqrt{\varepsilon_{i}}\right)
\end{array}\right],
$$

where $k$ is the wave number, $d_{i}$ is the thickness of the $i$-sublayer, $\varepsilon_{i}$ is the dielectric constant of the $i$-sublayer. The generalized matrix of the entire rough layer is represented as a product of intermediate matrices (7).

$$
M=\prod_{i=1}^{N} M_{i}=\left[\begin{array}{ll}
M_{11} & M_{12} \\
M_{21} & M_{22}
\end{array}\right] .
$$


From the general matrix, there is the reflection coefficient of the electromagnetic wave from the RS of PCM according to the formula (7) and the transmission coefficient according to the formula (8)

$$
\begin{aligned}
& \underline{R}=\frac{\left(M_{11}+M_{22}\right)-\left(M_{12}+M_{21}\right)}{M_{11}+M_{22}+M_{12}+M_{21}}, \\
& \underline{T}=\frac{2}{M_{11}+M_{22}+M_{12}+M_{21}} .
\end{aligned}
$$

Therefore, in order to accurately determine the plane of reflection from a rough surface, it is necessary to introduce a definition of $\varepsilon \Sigma$ to represent the phase characteristics of the RS through a multilayer structure. The introduction of the location of the plane of reflection from the RS makes it possible to compose a simpler mathematical model for describing the formation of the front of the reflected wave reflected from a smooth surface. That is, a smooth surface moves away or approaches the source of the sounding wave by a certain amount $\Delta$. Then the rough layer is expressed as a layer having a certain average value $\varepsilon$ over the layer. The RS of PCM varies from 1 micron to $0.5 \mathrm{~mm}$, the geometry depends on the manufacturing technology of the PCM (internal structure) and the method of machining. According to the analysis of PCM samples, it was revealed that: the range of values of $\underline{\varepsilon}$ ranges from 100 to 1500; The RS varies from 5 to 500 microns. On the basis of the multilayer structure of the RS, mathematical modeling was carried out to find the complex reflection coefficient $\underline{R}$ from different levels of the RS.

The obtained calculated dependencies make it possible to correct the measured value of the complex reflection coefficient. The procedure for using phase correction is carried out during the control of the measured sample of PCM with the RS using a traditional exemplary standard. The next step is to measure the profile of the RS by measuring the profilogram of the PCM sample, and using the profilometer, the level of the RS is determined. According to the measured data, the distribution and the level of the RS, the corresponding value of the phase shift is found, according to the value of which the result of the complex reflection coefficient from the PCM sample with the RS is refined. As a result, the value of the complex reflection coefficient from a PCM with a smooth surface is obtained. Then $\varepsilon$ is calculated. The result will be corrected and it will have reduced methodological uncertainty.

\section{References}

[1] Vasil'yev, E.N. Excitation of bodies of revolution., Radio i svyaz', Moscow, 1988.

[2] Kislyuk, M.ZH. The method of secondary waves in problems of electrodynamics Leningrad, 1970.

[3] Malushkov, G.D. Methods for solving problems of electromagnetic excitation of bodies of revolution., Izv. VUZov. Ser. Radiofizika. T.18(1975) 1563-1587.

https://doi.org/10.1007/BF01037102

[4] Nikol'skiy, V.V. Projection methods in electrodynamics., Vysshaya shkola, Moscow, 1977.

[5] Khizhnyak, N.A. Integral equations of macroscopic electrodynamics, Naukova Dumka, Kyiv, 1986. 
[6] Dmitrienko, G.V., Anisimov, V.G. Measurement of the permittivity of low-impedance composite materials at microwave frequencies., Measurement Techniquesthis, 52(2)(2009) 177183. https://doi.org/10.1007/s11018-009-9236-0

[7] GOST 27964-88. Measurement of roughness parameters., Izd-vo standartov, Moscow, 1989.

[8] Kornblit, S. Microwave optics. Optical principles in application to the design of microwave antennas. Svyaz', Moscow, 1980.

[9] Kuchin, A.A. Obradovich K.A. Optical devices for measuring surface roughness. Mashinostroyeniye, Leningrad, 1981.

[10]Baryshnikov I.V., Datskovskiy V.A., Upolovnev A.V. Vliyaniye sherokhovatoy poverkhnosti volnovedushchey sistemyna VCH-poteri. [Influence of the rough surface of the waveguide system on RF losses] / // Radio engineering and electronics. T.33(1988) 2029-2034.

[11] Danilov, YU.I. On the problem of microroughness of current-carrying surfaces in the microwave range of radio waves., Trudy LIAP. V.59 (1968), 92-96.

[12] Bogoroditskiy, N.P. Materials in radioelectronics., Gosenergoizdat, Moscow, 1975. 